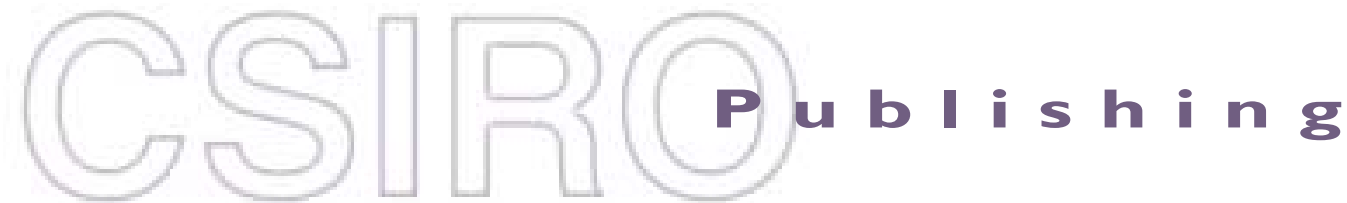

\section{Publications of the Astronomical Society of Australia}

Volume 19, 2002

(C) Astronomical Society of Australia 2002

An international journal of astronomy and astrophysics

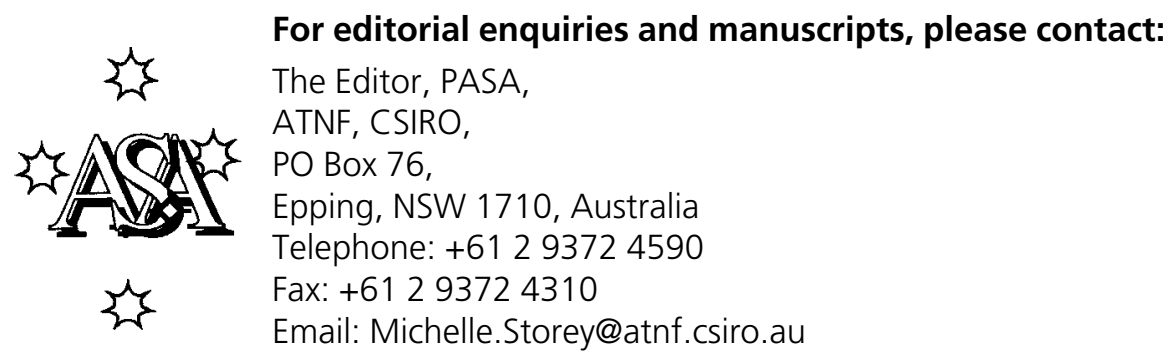

For general enquiries and subscriptions, please contact: CSIRO Publishing PO Box 1139 (150 Oxford St)

Collingwood, Vic. 3066, Australia

Telephone: +6139662 7666

Fax: +61 396627555

Email: publishing.pasa@csiro.au

C S I RO

PUBLISHING Published by CSIRO Publishing

for the Astronomical Society of Australia

www.publish.csiro.au/journals/pasa 


\title{
The Evolution of Shocks in Blazar Jets
}

\author{
Geoffrey V. Bicknell ${ }^{1,2}$ and Stefan J. Wagner ${ }^{3}$ \\ ${ }^{1}$ Research School of Astronomy and Astrophysics, Mt Stromlo Observatory, Cotter Road, \\ Weston, ACT 2611 \\ Geoff.Bicknell@anu.edu.au \\ ${ }^{2}$ Department of Physics and Theoretical Physics, ANU, Canberra, ACT 0200 \\ ${ }^{3}$ Landessternwarte, Koenigstuhl, D-69117 Heidelberg, Germany \\ S.Wagner@1sw.uni-heidelberg.de \\ Received 2002 January 16, accepted 2002 March 13
}

\begin{abstract}
We consider the shock structures that can arise in blazar jets as a consequence of variations in the jet flow velocity. There are two possible cases: (1) a double shock system consisting of both a forward and reverse shock, and (2) a single shock (either forward or reverse) together with a rarefaction wave. These possibilities depend upon the relative velocity of the two different sections of jet. Using previously calculated spherical models for estimates of the magnetic field and electron number density of the emission region in the TeV blazar Mkn 501, we show that this region is in the form of a thin disk in the plasma rest frame. It is possible to reconcile spectral and pair opacity constraints for Mkn 501 for Doppler factors in the range of 10-20. This is easiest if the corrections for $\mathrm{TeV}$ absorption by the infrared background are not as large as implied by recent models.
\end{abstract}

Keywords: acceleration of particles — BL Lacertae objects: general — BL Lacertae objects: individual (Mkn 501) — gamma rays: theory

\section{Introduction}

The current phenomenology used to estimate parameters in models of blazars often assumes a spherical homogeneous emitting region (in the plasma rest frame). This approximation is based in part on the ease of calculating the inverse Compton spectrum in a spherical geometry. This approach has served the subject well and has produced useful estimates of the parameters of many blazars (Ghisellini 1997). However, when we consider the reconciliation of constraints based upon spectral breaks and pair opacity, spherical models are probably too contrived. Furthermore, consideration of the way in which shocked regions would arise in jets led us away from the idea of spherical emitting regions. In this paper we take the first steps in the direction of determining the geometry of blazar emission and how this relates to the dynamics of the underlying flow. This indicates the direction for future blazar models and also indicates how models based upon more realistic geometries may be better able to constrain jet Doppler factors and the opacity of the diffuse infrared background.

\section{Spherical Models for Markarian 501}

Let us begin by noting the results obtained by fitting spherical emission models for the blazar Mkn 501. The geometry of such models is indicated in Figure 1. Typical parameters for Mkn 501, which emits synchrotron $\mathrm{X}$-rays and inverse Compton $\mathrm{TeV} \gamma$-rays, inferred from this geometry are a radius, $R \sim 10^{15}-10^{16} \mathrm{~cm}$, a magnetic field, $B \sim 0.1 \mathrm{G}$, magnetic energy, $U_{B} \sim 10^{-4} \mathrm{ergs} \mathrm{cm}^{-3}$, particle pressure, $\quad p \sim 10^{-2}-10^{0}$ dynes $\mathrm{cm}^{-2}$, and a Doppler factor, $\delta \sim 10-20$.

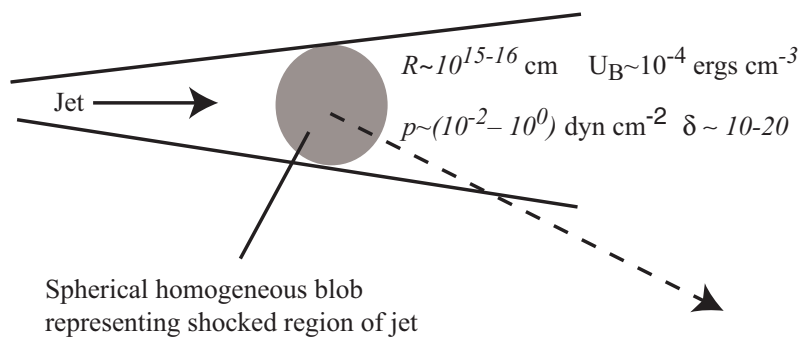

Synchrotron and inverse Compton radiation

Figure 1 A schematic indication of the spherical blob model for the emission region in blazar jets.

Mkn 501 flares sporadically in X-rays and $\gamma$-rays. Figure 2 shows the evolution of the $\mathrm{TeV} \gamma$-rays, $1 \mathrm{keV}$ RXTE PCA flux, and optical emission from Mkn 501 during part of a flare that occurred in July, 1997 (Lamer \& Wagner 1998). The period of observation encompasses a subsiding flare and a newly developing one, necessitating two component models for the flare. Note that the $1 \mathrm{keV}$ $X$-ray flux does not rise until about a day after the $\gamma$-ray flux. The reason for this is apparent in the time-dependent RXTE spectra (Lamer \& Wagner 1998) shown in Figure 3. Whilst the low energy part of the spectrum decreases, a high energy part starts to emerge at about epoch 4 and dominates in epoch 6. By epoch 10, the spectrum has returned to a form similar to that in epoch 2 exhibiting a break in spectral index of approximately 0.3 at around $6 \mathrm{keV}$.

A two component spherical model for the combined epoch $6 \mathrm{X}$-ray and epoch $6 \gamma$-ray data ${ }^{1}$ is shown in

${ }^{1}$ The $\gamma$-ray observations did not extend beyond epoch 6 because of increasing moonlight. 


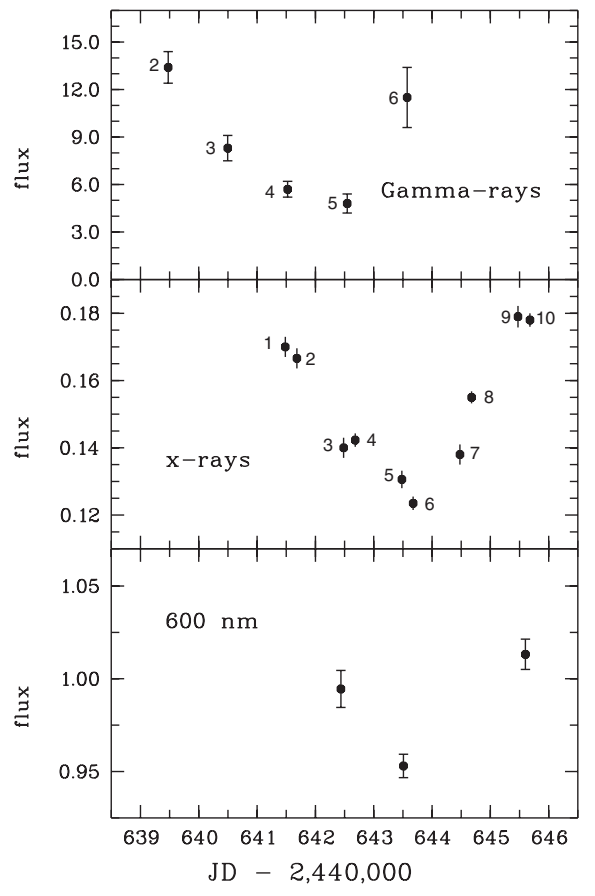

Figure 2 The integrated $\mathrm{TeV}$ count rate, the RXTE $1 \mathrm{keV}$ PCA count rate, and the variation in optical magnitude for the July, 1997 flare of Mkn 501 (Lamer \& Wagner 1998). Various epochs in the $\mathrm{TeV}$ and RXTE observations, referred to in the text, are indicated. The time of onset of the second flare is estimated (mainly from the RXTE spectra) to be at $t \approx 641.5$ days.

Figure 4. Two synchrotron components based on energytruncated power-law spectra were fitted to the X-ray data. We take the point of view that the high energy (break energy $\sim 50-100 \mathrm{keV}$ ) component whose emergence is apparent during earlier epochs is related to the $\gamma$-ray flare. The low energy component probably encompasses the lower energy X-rays from both the subsiding and developing flares. In order to restrict the number of parameters we have assumed that the magnetic field is the same in each component. The model parameters for each component are Doppler factor $\delta$, the upper cutoff in Lorentz factor of the electron distribution $\gamma_{2}$, the magnetic field (in the rest frame) $B$, the normalising constant of the electron distribution $K_{e}$, and the electron spectral index $a$. (The electron number density per unit Lorentz factor is $N(\gamma)=K_{e} \gamma^{-a}$.) The parameters reported in the figure legend relate only to the high energy component. The parameters of a number of model fits to this epoch of Mkn 501 are given in Table 1. These models include examples of where a correction has been made to the infrared background and examples of where no correction has been made. In relating the bulk Lorentz factor, $\Gamma$, to the Doppler factor we have assumed that the jet is nearly pole-on, so that $\delta \approx 2 \Gamma$. The bulk Lorentz factor is not used in the models but is used for other purposes below.

A typical fit to the Mkn 501 flux emitted during a large flare in July, 1997 is shown in Figure 4. In this fit, the $\mathrm{TeV}$ data have been corrected for absorption by the diffuse infrared background using the opacity estimated by
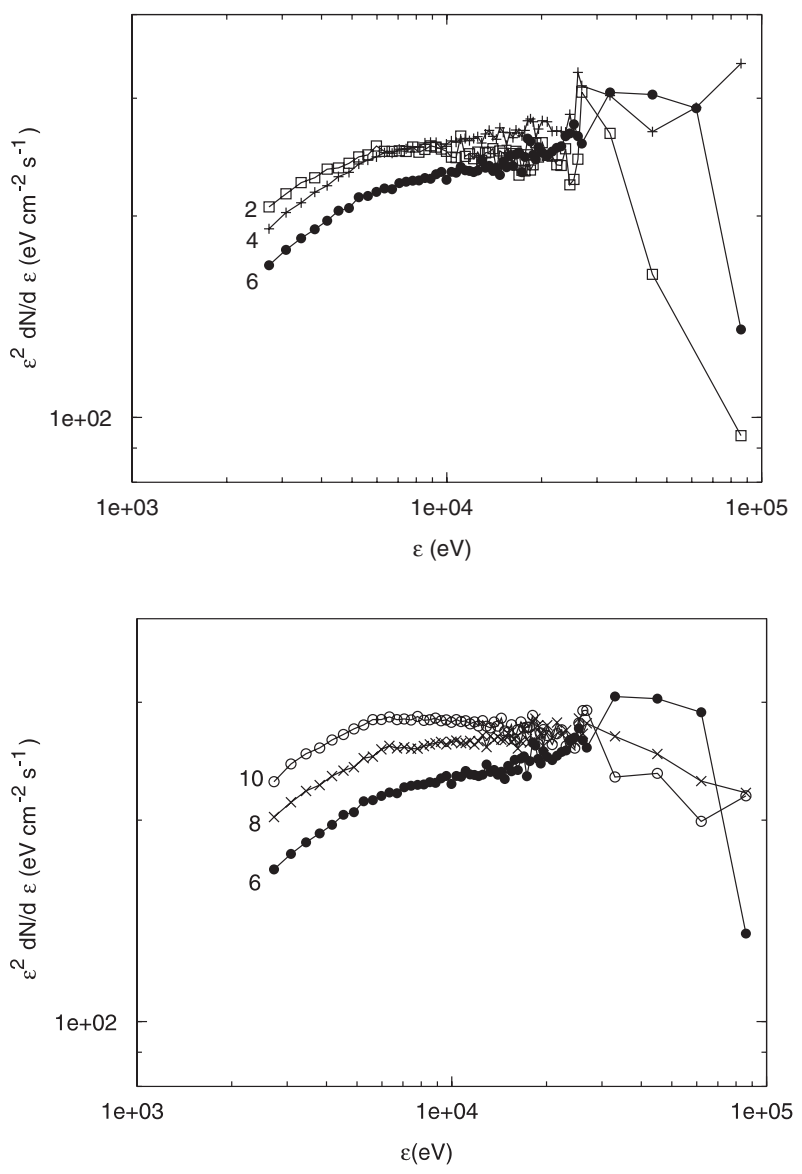

Figure 3 The development of the RXTE spectrum (Lamer \& Wagner 1998) over the period of the X-ray observations. The even epochs only are shown since these involved longer integration times than the odd numbered epochs providing adequate signal to noise in the 20-100 keV range. Error bars have been left off in order to distinguish the different spectra.

Guy et al. (2000). However, the extent of the correction is currently controversial and we have also summarised in Table 1 the model parameters derived when no correction is made. It is worth emphasizing that the inferred radius of the spherical region is a parameter that is provided by the model fits. Variability provides an a priori upper limit on this parameter, namely that for a variability timescale, $\Delta t$, the emitting region needs to be small enough that variations are not smeared out. This leads to

$$
R<\frac{1}{2} c \Delta t \delta \approx 1.3 \times 10^{16}\left(\frac{\Delta t}{\text { day }}\right)\left(\frac{\delta}{10}\right) \mathrm{cm} .
$$

However, in the spherical model, there is no other a priori basis for radii $\sim 10^{15}-10^{16} \mathrm{~cm}$. Sometimes the diameter of the sphere is associated with an adiabatic cooling length. However, this idea has not really been developed significantly.

\section{Production of Shocks in Jets}

In order to make further progress it is important to understand the possible processes that can lead to the production of shocks in jets. One process that is often mentioned (e.g. Rees 1978) is the production of shocks resulting from 


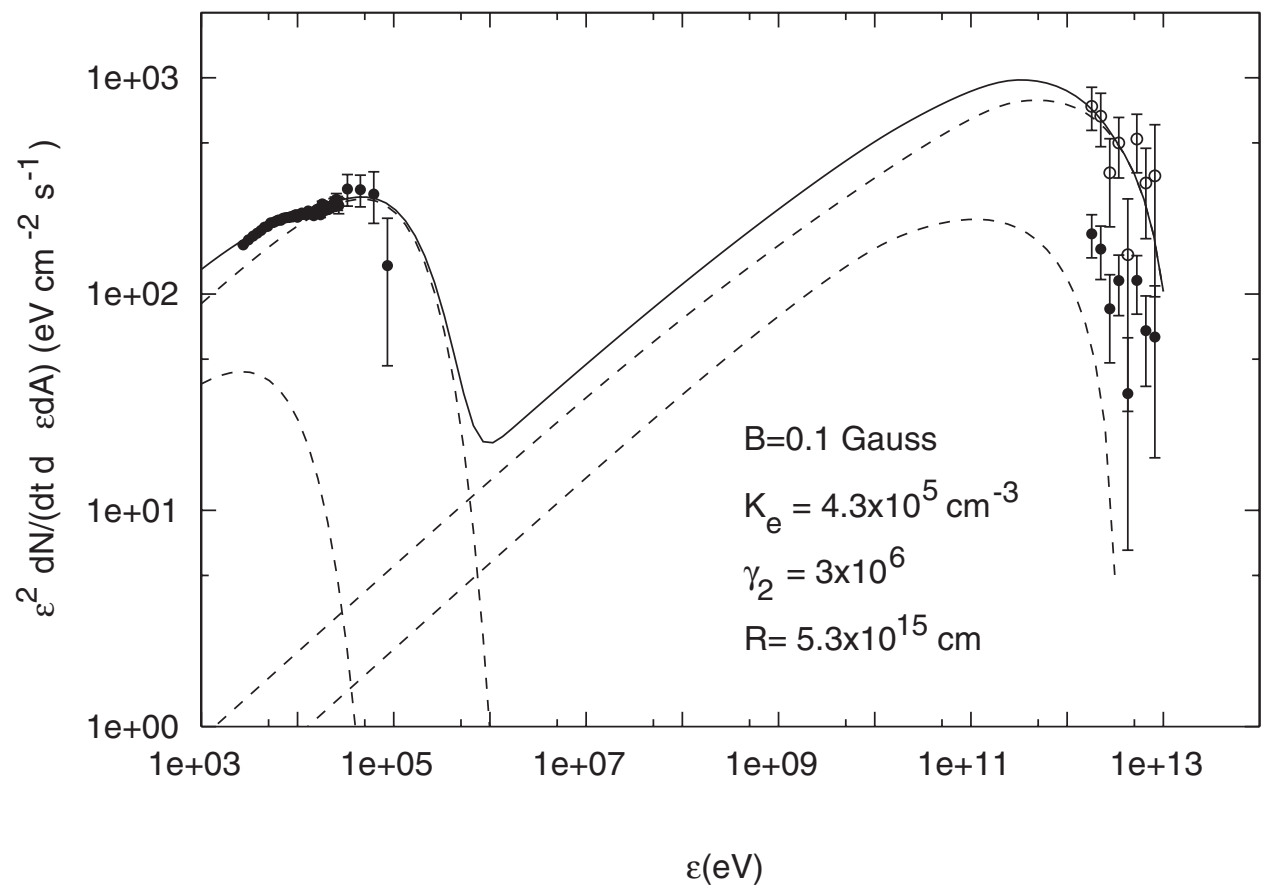

Figure 4 A fit to the combined epoch $6 \mathrm{X}$-ray and epoch $6 \mathrm{TeV} \gamma$-ray data from the July, 1997 flare from Mkn 501 assuming a Doppler factor of 10 (Bicknell, Wagner, \& Groves 2001). The TeV data have been corrected using the opacity of the IR background derived by Guy et al. (2000). The raw data are shown as solid circles; the corrected data are shown as open circles. However, the extent of the required correction is currently uncertain.

Table 1. Comparison of parameters of synchrotron plus inverse Compton model fit to data on Mkn 501. The emitting region is assumed to be spherical in the rest frame

\begin{tabular}{|c|c|c|c|c|c|c|c|c|c|}
\hline Model & $\delta$ & $\Gamma$ & $\gamma_{2}$ & $a$ & $\begin{array}{c}B \\
\text { (Gauss) }\end{array}$ & $\begin{array}{c}K_{e} \\
\left(\mathrm{~cm}^{-3}\right)\end{array}$ & $\begin{array}{c}p_{e} \\
\left(\text { dynes cm } \mathrm{cm}^{-2} \text { ) }\right.\end{array}$ & $\begin{array}{c}u_{B} \\
\left(\operatorname{ergs~cm} \mathrm{cm}^{-3}\right)\end{array}$ & $\begin{array}{c}R \\
\left(10^{15} \mathrm{~cm}\right)\end{array}$ \\
\hline \multicolumn{10}{|c|}{ IR background corrected } \\
\hline 1 & 10 & 5 & $3 \times 10^{6}$ & 2.0 & 0.1 & $4.3 \times 10^{5}$ & 1.2 & $4.0 \times 10^{-4}$ & 5.3 \\
\hline 2 & 20 & 10 & $1.5 \times 10^{6}$ & 2.0 & 0.2 & $2.6 \times 10^{6}$ & 6.8 & $1.6 \times 10^{-3}$ & 0.87 \\
\hline \multicolumn{10}{|c|}{ Not corrected for IR background } \\
\hline 3 & 10 & 5 & $3 \times 10^{6}$ & 2.0 & 0.06 & $4.4 \times 10^{3}$ & 0.012 & $1.4 \times 10^{-4}$ & 14 \\
\hline 4 & 20 & 10 & $1.5 \times 10^{6}$ & 2.0 & 0.13 & $5.4 \times 10^{4}$ & 0.14 & $6.7 \times 10^{-4}$ & 1.5 \\
\hline
\end{tabular}

variations in the flow velocity. We idealise the initial conditions as a velocity discontinuity with faster moving gas catching up to slower moving gas (see Figure 5). This is a classic shock tube in which the equation of state and flow velocities are relativistic. There are two solutions of interest: (1) Two shock waves are produced, one moving into the gas ahead of the shock - the forward shock, the other into the gas behind - the reverse shock. (2) A single shock (either forward or reverse) and a rarefaction are produced. The direction of the shock in this case depends upon the details of the initial parameters. The development of the two possible solutions is sketched in Figure 6.

Both of these situations are most readily analysed by transforming to the frame of the contact discontinuity separating the two gases. This is the approach used by Landau \& Lifshitz (1987) for the non-relativistic case. It is also useful here since, as before, this is the rest frame of the two gases on either side of the contact discontinuity and is the appropriate frame in which to calculate the emissivity.

\subsection{Forward and Reverse Shocks}

Let us first consider the case of a forward and reverse shock. It is useful to calculate the difference in the velocities of the two shocks given the (uniform) pressure, $p_{3}$, of the gas sandwiched in between them. In so doing we use the ultrarelativistic equation of state, $p=1 / 3 \epsilon$, where $p$ is the pressure and $\epsilon$ is the energy density. This is equivalent to assuming that at this stage of the jet, some 100 gravitational radii from the core, there is no entrained thermal matter and that the initial matter ejected from the black hole is ultrarelativistic (e.g. is composed exclusively of relativistic electron-positron pairs). The most straightforward way to do the calculation is in the 
frame of the contact discontinuity (CD) separating the two shocked regions, assign the ratio $p_{3} / p_{1}$, and then use this to calculate velocities of both shocks and the velocities of upstream and downstream gas.

In the frame of the $\mathrm{CD}$, the velocities of upstream and downstream gas in regions 1 and 2 may be derived from the expressions given in Landau \& Lifshitz (1987) for the relative velocity between pre-shock and post-shock gases, taking into account that in the $\mathrm{CD}$ frame the post-shock gas is stationary. The result is:

$$
\begin{gathered}
\beta_{1}^{\prime}=\frac{\sqrt{3}\left(p_{3} / p_{1}-1\right)}{\sqrt{\left(3+p_{3} / p_{1}\right)\left(1+3 p_{3} / p_{1}\right)}} \\
\beta_{2}^{\prime}=-\frac{\left(p_{3} / p_{1}-p_{2} / p_{1}\right)}{\sqrt{\left(3 p_{2} / p_{1}+p_{3} / p_{1}\right)\left(p_{2} / p_{1}+3 p_{3} / p_{1}\right)}} .
\end{gathered}
$$

At this point we introduce a convenient notation for the relativistic composition of velocities. We denote the

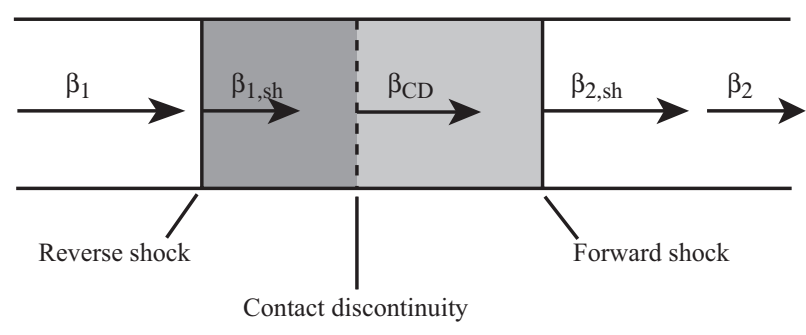

Figure 5 The production of forward and reverse shocks in a jet resulting from variations in the flow velocity. Note that the relative transverse and longitudinal sizes are not to scale. Modelling shows that the longitudinal extent is generally much smaller than the radial extent (see Table 2). relativistic addition and subtraction of velocities by

$$
\beta_{1} \oplus \beta_{2} \stackrel{\text { def }}{=} \frac{\beta_{1}+\beta_{2}}{1+\beta_{1} \beta_{2}}
$$

and

$$
\beta_{1} \ominus \beta_{2} \stackrel{\text { def }}{=} \frac{\beta_{1}-\beta_{2}}{1-\beta_{1} \beta_{2}}
$$

respectively.

The frame-independent relative velocity between the gas on either side of the forward/reverse shock structure

$$
\begin{aligned}
\beta_{12} & =\beta_{1} \ominus \beta_{2} \\
& =\beta_{1}^{\prime}\left(p_{3} / p_{1}, p_{2} / p_{1}\right) \ominus \beta_{2}^{\prime}\left(p_{3} / p_{1}, p_{2} / p_{1}\right)
\end{aligned}
$$

may be used to either calculate the relative velocity in the observer's frame as a function of the parameters $p_{3} / p_{1}$ and $p_{2} / p_{1}$ or to numerically determine the ratio $p_{3} / p_{1}$ of the shock to upstream pressures as a function of the relative velocity and the ratio $p_{2} / p_{1}$ of the downstream to upstream pressures.

What is most important for the current purposes is the difference in the shock velocities since this determines the size of the emitting region in the rest frame. These may be calculated from the expressions given in Landau \& Lifshitz (1987) for the pre-shock and post-shock velocities in the shock frame. Denoting the shock frame by a double prime, then the velocity of the reverse shock, for example, in the $\mathrm{CD}$ frame, is given by:

$$
\beta_{1}^{\prime} \ominus \beta_{1, \mathrm{sh}}^{\prime}=\beta_{1}^{\prime \prime} \Rightarrow \beta_{1, \mathrm{sh}}^{\prime}=\beta_{1}^{\prime} \ominus \beta_{1}^{\prime \prime}
$$
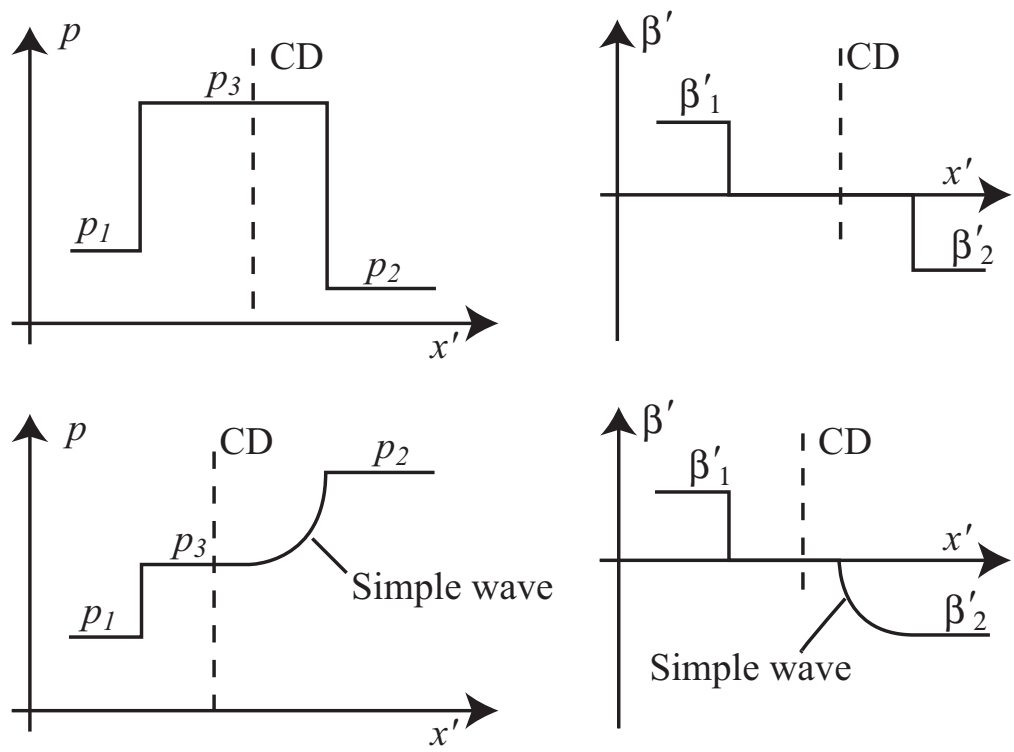

Figure 6 The behaviour of pressure and velocity in a relativistic shock tube. The upper part of the diagram is for the case wherein a forward and reverse shock form; the lower part of the diagram is for the case of a reverse shock plus a relativistic simple wave. The frame of the contact discontinuity is also the rest frame of the emitting plasma. 
The results for both forward and reverse shocks are:

$$
\begin{aligned}
& \beta_{1, \mathrm{sh}}^{\prime}=-\sqrt{\frac{3+p_{3} / p_{1}}{3\left(1+3 p_{3} / p_{1}\right)}} \\
& \beta_{2, \mathrm{sh}}^{\prime}=\sqrt{\frac{3+p_{3} / p_{2}}{3\left(1+3 p_{3} / p_{2}\right)}} .
\end{aligned}
$$

The limits of strong and weak shocks are informative.

$$
\begin{array}{ll}
\text { As } \frac{p_{3}}{p_{1}} \rightarrow \infty & \beta_{1, \mathrm{sh}}^{\prime}, \beta_{2, \mathrm{sh}}^{\prime} \rightarrow \mp \frac{1}{3} \\
\text { As } \frac{p_{3}}{p_{1}} \rightarrow 1 & \beta_{1, \mathrm{sh}}^{\prime}, \beta_{2, \mathrm{sh}}^{\prime} \rightarrow \mp \frac{1}{\sqrt{3}} .
\end{array}
$$

These limits are to be expected from the limiting velocities in the post-shock region of a relativistic flow, remembering that the post-shock region is at rest in this frame. Note also that the range in velocities between strong and weak shocks is not great - approximately $0.33-0.58 c$. The shock velocity difference, which determines the size of the emitting region, is shown in Figure 7. Note that the size of the region is not determined by the relative velocity. However, the relative velocity has a role which we consider further below.

The size of the flaring region in the rest frame as a function of time in the rest frame, $\Delta t^{\prime}$, and the observer's time, $\Delta t$, is given by

$$
\begin{aligned}
\Delta x_{\mathrm{sh}}^{\prime}= & \left|\beta_{1, \mathrm{sh}}^{\prime}-\beta_{2, \mathrm{sh}}^{\prime}\right| c \Delta t^{\prime}=\frac{\left|\beta_{1, \mathrm{sh}}^{\prime}-\beta_{2, \mathrm{sh}}^{\prime}\right|}{\Gamma_{\mathrm{CD}}} c \Delta t \\
\approx & 2.6 \times 10^{14}\left|\beta_{1, \mathrm{sh}}^{\prime}-\beta_{2, \mathrm{sh}}^{\prime}\right| \\
& \times\left(\frac{\Gamma_{\mathrm{CD}}}{10}\right)^{-1}\left(\frac{t}{1 \text { day }}\right) \mathrm{cm} .
\end{aligned}
$$

Time dilation between the rest and observer's frame has been incorporated. Since the difference in shock velocities does not vary greatly with the pressure of the shocked gas, we adopt a fiducial value of unity for that parameter.

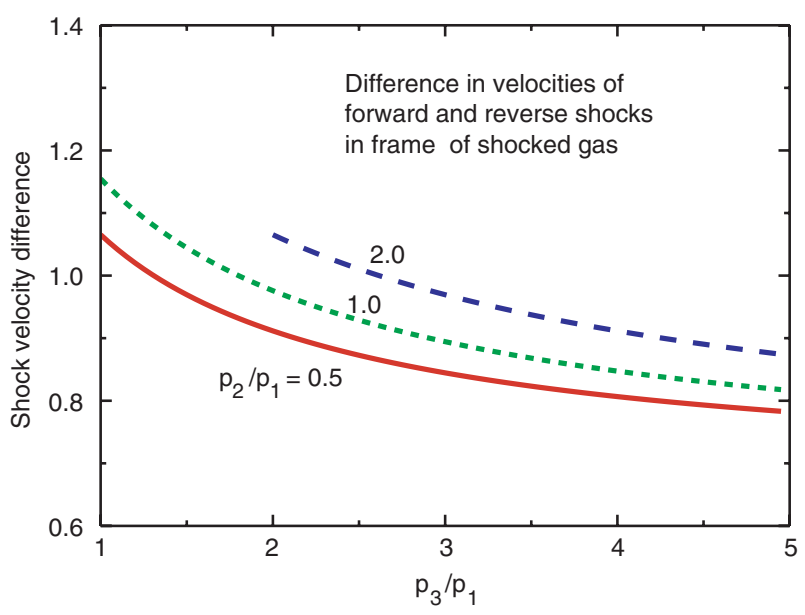

Figure 7 The difference in velocities of forward and reverse shocks in the case when two shocks are produced.
An immediately interesting feature of this result is that for a Lorentz factor of 5 (corresponding to a Doppler factor $\sim 10$ ) and timescales of a few days, the size of the shocked region in the direction of flow is comparable to the radii inferred from fits of the spherical model. This is a direct consequence of the insensitivity of the size of the shocked region to its pressure.

\subsection{The Relative Velocity and the Condition for Two Shocks}

We have already seen that the pressure in the shocked region can be estimated from the relative velocity of the unshocked gas on either side of the reverse-forward shock region. The relative velocity also enters into the condition for a forward-reverse shock pair to occur. The relative velocity needs to be large enough to produce a pressure, $p_{3}$, between the shocks that is greater than both $p_{1}$ and $p_{2}$. The limiting case occurs when $p_{3}=\max \left(p_{1}, p_{2}\right)$. This leads to the following condition on the relative velocity, $\beta_{12}$, of the gas on either side of the shocked region:

$$
\beta_{12}>\sqrt{3} \frac{\left|p_{2} / p_{1}-1\right|}{\sqrt{\left(3 p_{2} / p_{1}+1\right)\left(3+p_{2} / p_{1}\right)}} .
$$

If the relative velocity is less than this critical value, that is, if the variation in velocity is not substantial enough, then a forward-reverse shock structure does not form. In order to gain some numerical insight, one can assign a Lorentz factor to the overtaking gas (region 1) and use equation (13) to calculate the maximum Lorentz factor of the gas ahead of the forward shock (region 2) for a twoshock solution to occur. The maximum Lorentz factor is shown in Figure 8 for overtaking Lorentz factors, $\Gamma_{1}$, of 5 and 10 . The relevant parameter here is the ratio of the pressures downstream of the forward shock, $p_{2}$, and upstream of the reverse shock, $p_{1}$. For example, if $p_{2} / p_{1}=2$ and $\Gamma_{1}=5$ then the maximum Lorentz factor of the upstream

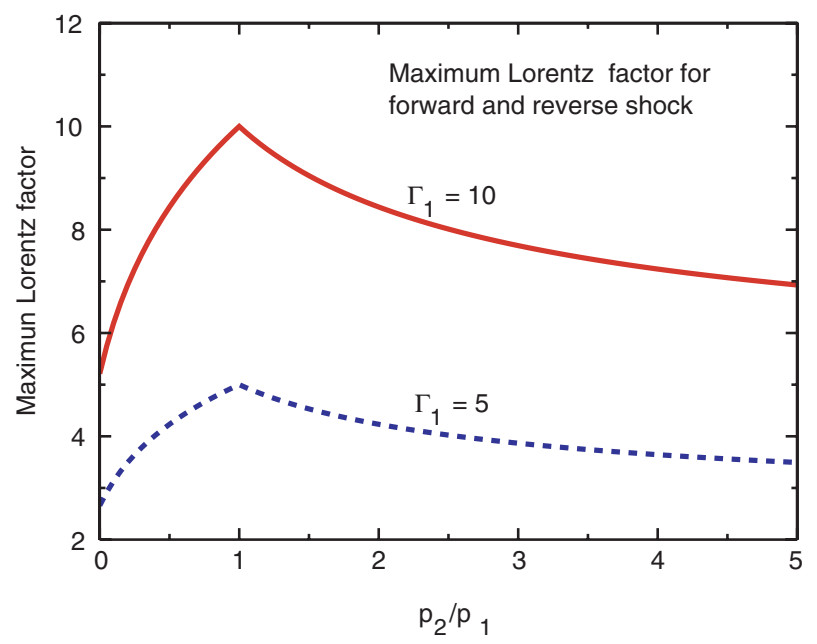

Figure 8 This plot shows the maximum Lorentz factor of the gas being overtaken if both a forward and reverse shock are to result. The maximal Lorentz factors are shown for two values, 5 and 10, of the Lorentz factor of the overtaking gas. 
gas is about 4.3. If the Lorentz factor of the upstream gas exceeds this value then an intermediate shocked region with pressure greater than both $p_{2}$ and $p_{1}$ cannot be supported. The outcome is a rarefaction wave moving into the downstream gas and a reverse shock moving into the upstream gas. When $p_{1}>p_{2}$ the situation is reversed.

As in the non-relativistic case (see Landau \& Lifshitz 1987), the rarefaction region is described by a centred, simple wave (Liang 1977). Simple waves are based on the Riemann invariants, $J_{ \pm}$, of the one-dimensional gas dynamics equations and for the equation of state $p=\epsilon / 3$

$$
J_{ \pm}=\frac{1}{2} \ln \frac{1+\beta}{1-\beta} \pm \frac{\sqrt{3}}{4} \ln p .
$$

In the region of the right propagating simple wave, $J_{-}=$constant and the equations of the right propagating characteristics are

$$
\frac{x}{c t}=\frac{\beta+1 / \sqrt{3}}{1-\beta / \sqrt{3}} .
$$

These two features of a centred simple wave allow one to develop the solution in the rarefaction region and patch it to the solution on the left representing the reverse shock (see Figure 6). As with the two-shock solution it is convenient to develop the shock plus rarefaction solution in the frame of the $\mathrm{CD}$ and in terms of the pressure, $p_{3}$, in the postshock region. In this frame, the unshocked gas velocity on the left is given by

$$
\beta_{1}^{\prime}=\frac{\sqrt{3}\left(p_{3} / p_{1}-1\right)}{\sqrt{\left(3+p_{3} / p_{1}\right)\left(1+3 p_{3} / p_{1}\right)}}
$$

and the velocity on the right is given by

$$
\beta_{2}^{\prime}=\frac{\left(p_{2} / p_{3}\right)^{\sqrt{3} / 2}-1}{\left(p_{2} / p_{3}\right)^{\sqrt{3} / 2}+1} .
$$

The frame-independent relative velocity, $\beta_{12}=\beta_{1}^{\prime} \ominus \beta_{2}^{\prime}$, can be used to solve numerically for the pressure, $p_{3}$, in terms of $\beta_{12}$ if required.

In this solution, the velocity of the reverse shock in the $\mathrm{CD}$ frame (which is also the rest frame of the shocked gas) is the same as that of the reverse shock in the two-shock case, i.e.

$$
\beta_{1}^{\prime}=-\sqrt{\frac{3+p_{3} / p_{1}}{3\left(3 p_{3} / p_{1}+1\right)}}
$$

and the size of the shocked region in the CD frame as a function of the observer's time is also similar, viz.:

$$
\Delta x^{\prime}=\left|\beta_{1}^{\prime}\right| \frac{c \Delta t}{\Gamma} \approx 2.6 \times 10^{14}\left|\beta_{1}^{\prime}\right|\left(\frac{\Gamma}{10}\right)^{-1}\left(\frac{t}{\text { day }}\right) \mathrm{cm} \text {. }
$$

\section{Application to the Flare in Markarian 501 - the Size of the Shocked Region}

Inspection of the RXTE spectra and the $\gamma$-ray light curve shows that the second flare begins at $t \approx 641.5$ days where $t$ refers to JD-2,440,000. This means that epoch 6 for which we have presented the two component models (see Figure 4) corresponds to $\Delta t \approx 643.7-641.5=2.2$ days and X-ray epoch 10 corresponds to $\Delta t \approx 645.7-$ $641.5=4.2$ days. The entire period surrounding this flare involved such a marked departure from the quiescent flux of Mkn 501 that it is reasonable to assume that the relative velocity exceeded the critical value derived above and that $\left|\beta_{1, \mathrm{sh}}-\beta_{2, \mathrm{sh}}\right| \sim 1$ giving a longitudinal size for the flaring region $\sim 1.1 \times 10^{15}(\Gamma / 5)^{-1} \mathrm{~cm}$. We neglect for now the complications resulting from calculating the inverse Compton emissivity in a non-spherical region and, in order to obtain indicative estimates of the radius of the emitting disk-like region of the jet, we assume that the volume of the emitting region is the same as that of the corresponding spherical region as modelled previously. As recorded in Table 2, this gives radii ranging from about $9 \times 10^{14} \mathrm{~cm}$ to $4 \times 10^{16} \mathrm{~cm}$. Thus, the emitting region is a reasonably thin slice of the jet, and in the rest frame, the ratio of its longitudinal extent to its diameter ranges from 0.01 to 0.3 , depending upon the details of the model. The estimate of the longitudinal size arises naturally from the dynamics of the shocked region and one does not have to appeal to adiabatic expansion to limit the effective size. This is also the case for the shock plus rarefaction solution.

\section{Constraints from Spectral Breaks}

As the flare evolves, by epoch 10 the X-ray spectrum (see Figure 3 has settled down to a shape similar to that of the original flare in epoch 2 . We regard this as indicating the establishment of a quasi-steady state spectrum in which cooling following a shock (or shocks) has established a broken power law with the break energy at about $6 \mathrm{keV}$. This spectral feature provides further constraints on the model with interesting implications.

Table 2. Indicative parameters for the disk model based upon the approximations described in the text

\begin{tabular}{lcccccccc}
\hline Model & $\begin{array}{c}\Delta l^{\prime} \\
\text { Epoch 6 } \\
10^{15} \mathrm{~cm}\end{array}$ & $\begin{array}{c}\Delta l^{\prime} \\
\text { Epoch } 10 \\
10^{15} \mathrm{~cm}\end{array}$ & $\begin{array}{c}\text { Radius } \\
\text { of disk } \\
10^{15} \mathrm{~cm}\end{array}$ & $\begin{array}{c}u_{\text {rad }}^{\prime} / u_{B} \\
\text { (n) }\end{array}$ & $\begin{array}{c}\Delta t_{c} \\
\text { Epoch 10 } \\
\text { days }\end{array}$ & $\begin{array}{c}\Delta t_{c} / \Delta t \\
\text { Epoch 10 }\end{array}$ & $\begin{array}{c}\tau_{\gamma \gamma} \\
\text { Diameter } \\
10 \mathrm{TeV}\end{array}$ & $\begin{array}{c}\tau_{\gamma \gamma} \\
\Delta l^{\prime} \\
10 \mathrm{TeV}\end{array}$ \\
\hline 1 & 1.1 & 2.2 & 9.5 & 4.9 & 1.3 & 0.31 & 3.3 & 0.20 \\
2 & 0.57 & 1.1 & 0.90 & 8.5 & 0.80 & 0.19 & 1.2 & 0.39 \\
3 & 1.1 & 1.1 & 4.1 & 0.95 & 8.4 & 2.0 & 1.1 & 0.015 \\
4 & 0.57 & 2.2 & 2.0 & 5.3 & 2.3 & 0.55 & 0.15 & 0.11 \\
\hline
\end{tabular}


The criterion for the location of the break is somewhat contrived in the framework of a spherical model. This is especially the case with temporal data since there is really no criterion for how the radius of the emitting region would evolve. We therefore shall not burden the reader with calculations appropriate for a spherical geometry and we only consider the implications of the spectral break for the disk model whose dynamics we have considered above.

A break in spectral index in the context of shockgenerated spectra occurs at that photon energy where the cooling time of electrons is equal to the travel time of the plasma across the emitting region. In this case this means that the cooling time at the break energy equals the duration of the flare. Often, only the effect of synchrotron cooling is considered. However, in many blazars the inverse Compton power is comparable to or greater than the synchrotron power and the contribution of inverse Compton cooling to the total cooling timescale must be considered. When inverse Compton scattering in the Klein-Nishina limit is involved (as it is for $\mathrm{TeV}$ blazars) the calculation of the cooling time is more complicated than it is when the Thomson limit applies. However, in the Klein-Nishina limit, both the cross-section and energy transfer decrease and we therefore assume that the dominant inverse Compton contribution occurs for interactions between photons and the radiation field that occur in the Thomson limit. Thus, in estimating the appropriate radiation energy density for an electron of Lorentz factor, $\gamma$, in the plasma rest frame, one determines a cutoff photon energy, $\epsilon_{2}$, in the observer's frame, defined by

$$
\epsilon_{2}(\gamma) \approx \delta \frac{m_{e} c^{2}}{\gamma} \approx 5.1\left(\frac{\delta}{10}\right)\left(\frac{\gamma}{10^{6}}\right)^{-1} \mathrm{eV} .
$$

For a cylindrical uniformly emitting disk of length, $\Delta l$, and radius, $R$, the radiation energy density per unit frequency at the centre of the disk in the plasma rest frame and the observed flux density are given by

$$
\begin{aligned}
& u_{v}^{\prime}=\frac{4 \pi j_{v^{\prime}}^{\prime}}{c} f\left(\Theta_{c}\right) \\
& F_{v}=\frac{\delta^{3}}{D_{L}^{2}} \pi R^{2} \Delta l j_{v^{\prime}}^{\prime}
\end{aligned}
$$

where

$$
\Theta_{c}=\tan ^{-1}(\Delta l / 2 R)
$$

and

$$
f\left(\Theta_{c}\right)=\Theta_{c}-\tan \Theta_{c} \ln \sin \Theta_{c} .
$$

For a thin disk, $\Theta_{c} \approx \Delta l / 2 R$ and $f\left(\Theta_{c}\right) \approx(\Delta l / 2 R) \times$ $(1+\ln (2 R / \Delta l))$. Thus the radiation energy density is defined in terms of the flux density, $F_{\epsilon}=$

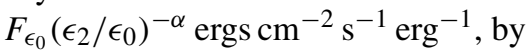

$$
u_{\mathrm{rad}}^{\prime} \approx \frac{4 D_{L}^{2}}{c R \Delta l} f\left(\Theta_{c}\right) \delta^{-4}\left(\frac{\epsilon_{0} F_{\epsilon_{0}}}{1-\alpha}\right)\left(\frac{\epsilon_{2}}{\epsilon_{0}}\right)^{1-\alpha}
$$

where $\epsilon_{2}(\gamma)$ is the cutoff in photon energy (observer's frame) defined above and $\epsilon_{0}=1 \mathrm{keV}$ is a convenient fiducial energy.
The synchrotron plus inverse Compton cooling time, corresponding to the break energy, $\epsilon_{b}$, is, in the plasma rest frame

$$
\begin{aligned}
\Delta t_{c}^{\prime}= & 2^{1 / 2} 3^{3 / 2} \pi \frac{\left(m_{e} c e \hbar\right)^{1 / 2}}{\sigma_{T}} B^{-3 / 2}\left[1+\frac{u_{\mathrm{rad}}}{u_{B}}\right]^{-1} \epsilon_{b}^{\prime-1 / 2} \\
\approx & 3.23 \times 10^{3} B^{-3 / 2}\left[1+\frac{u_{\mathrm{rad}}^{\prime}}{u_{B}}\right]^{-1} \\
& \times \delta^{1 / 2}\left(\frac{\epsilon_{b}}{\mathrm{keV}}\right)^{-1 / 2} \mathrm{sec}
\end{aligned}
$$

where $u_{B}=B^{2} / 8 \pi$. In order to compare with the observed flare duration, we use the corresponding time in the observer's frame:

$$
\begin{aligned}
\Delta t_{c}= & \Gamma t_{c}^{\prime} \approx 3.23 \times 10^{3} B^{-3 / 2}\left[1+\frac{u_{\mathrm{rad}}}{u_{B}}\right]^{-1} \\
& \times\left(\delta \Gamma^{2}\right)^{1 / 2}\left(\frac{\epsilon_{b}}{\mathrm{keV}}\right)^{-1 / 2} \mathrm{sec} .
\end{aligned}
$$

Normally, one would invert the expression for $\Delta t_{c}$ to obtain an expression for the break energy $\epsilon_{b}$ as a function of time. However, $\Delta t_{c}$ depends both explicitly on the break energy and implicitly through the dependence of $u_{\text {rad }}$ upon $\epsilon_{b}$. Therefore, in assessing the implications of the break in the X-ray spectrum, it is easiest to compare the cooling time, $\Delta t_{c}$, at the observed break energy with the time of duration of the flare (approximately 4.2 days at epoch 10). These comparisons are recorded in Table 2 for the various models summarised in Table 1.

\section{Pair Opacity}

Pair opacity provides another important constraint on models of blazars - particularly TeV blazars. TeV photons interact with infrared photons to produce pairs, the rate of production again depending upon the radiation energy density in the plasma rest frame. Here we are concerned with the infrared photons internal to the emitting region. For a power-law photon spectrum of spectral index $\alpha$ extending to at least the infrared, the absorption per unit length, $a_{\gamma \gamma} \mathrm{cm}^{-1}$, of a $\gamma$-ray of energy $\epsilon_{\gamma}$ can be expressed in terms of the observed flux density $F_{\epsilon_{0}}$, and observed $\gamma$-ray energy $\epsilon_{\gamma}$, using results presented in Svensson (1987), as follows:

$$
\begin{aligned}
a_{\gamma \gamma}= & \frac{4 \sigma_{T}}{c} \eta(\alpha) \frac{d_{L}^{2}}{\Delta l R} f\left(\Theta_{c}\right) \delta^{-(2 \alpha+3)} \\
& \times F_{\epsilon_{0}}\left[\frac{\epsilon_{0} \epsilon_{\gamma}}{\left(m_{e} c^{2}\right)^{2}}\right]^{\alpha} \mathrm{cm}^{-1} .
\end{aligned}
$$

An analytical expression for the parameter $\eta(\alpha) \sim 1$ has been calculated by Svensson (1987). The opacity depends upon the Doppler factor to a large power, so that a large Doppler factor can also render the observation of $\mathrm{TeV} \gamma$-rays consistent with the photon energy densities required to produce them. 
At first sight it appears that this disk model for the shocked region offers a better prospect for the escape of $\gamma$-rays because of the reduced path length in the direction of the jet. However, aberration implies that in the rest frame, photon trajectories are almost perpendicular to the jet thereby increasing the path length. Specifically, if $\Delta l^{\prime}$ is the size, in the rest frame, of the disk, if $\theta$ is the angle between the jet and the line of sight in the observer's frame, and if $c \beta$ is the jet velocity, then the path length of a ray through the disk originating from the back is given by

$$
\frac{\Delta s}{\Delta l^{\prime}}=\frac{1-\beta \cos \theta}{\cos \theta-\beta}
$$

(with the proviso that $\Delta L$ has a maximum $\sim$ the diameter of the shocked region). The value of $\Delta s / \Delta l^{\prime}$ tends to compensate for the smaller value of $\Delta l^{\prime}$ implied by our disk model so that one can only expect a modest reduction in $\gamma$-ray opacity compared to a spherical model. Therefore in Table 2 we have given the $\gamma$-ray opacity for $10 \mathrm{TeV}$ photons based on the diameter of the emitting disk. The emitting region is only marginally optically thin in this case.

On the other hand, the specific normal shock configuration, upon which we have based our models, represents the worst case for $\gamma$-ray opacity. If the shock(s) defining the disk were oblique then the photons emitted perpendicular to the jet direction in the rest frame would not have such a large path length and a reduction in opacity by an order of magnitude would result. We have, therefore, as an indication of the reduction in opacity related to this model, tabulated the optical depth based upon the longitudinal extent of the slab. Naturally this is much less and indicates the importance of generalising this model to the case of oblique shocks.

Despite these potential reductions in $\gamma$-ray opacity, it appears that the emission of $\gamma$-rays originates not far above the $\tau_{\gamma \gamma}=1$ surface - the gamma-sphere (Blandford 1994; Blandford \& Levinson 1995). It is possible, therefore, that X-ray flares generated at smaller radii will have no $\mathrm{TeV}$ counterpart. An examination of the combined $\mathrm{TeV}$ and X-ray light curves would be interesting in this regard.

\section{Discussion}

\subsection{Summary}

We have presented a simple theoretical approach to determining the geometry of synchrotron and inverse Compton emitting regions in blazar jets that is intimately related to the dynamics of the underlying flow. Of course, other treatments of blazar emission regions that postulate relativistic shocks from the outset (e.g. Mastichiadis, Georganopoulos, \& Kirk 2001) would give similar estimates for the size of the emitting region. What we have shown here is how the emission region patches onto the rest of the jet and how it is determined by the flow parameters of different sections. For high enough relative velocities, the emission region is bounded by two shocks; for relative velocities lower than critical the emission region patches on via a simple wave. In the latter case both forward and reverse shocks are possible depending upon the relative strengths of the pressure in the two regions.

Using this approach, we have shown that the extent (in the jet direction) of the shocked emission region is similar to, but smaller than, what has previously been inferred from spherical models. Making the approximation that the inverse Compton emission from a shocked section of jet is the same as from the equivalent sphere, we have shown how spectral breaks and pair optical depths may be estimated. There are some interesting differences here between models calculated using $\mathrm{TeV}$ spectra that have been corrected to allow for the opacity of the infrared background (IRB) and comparable models for which no corrections have been applied. Referring to Table 2, one can see that the IRB-corrected models are more compact and the estimated cooling times are less than the observed duration of the flare. Moreover, the pair opacity at $10 \mathrm{TeV}$ is quite significant even in the most favourable case of basing the opacity on the length of the shocked region. In the case of no IRB correction, it is feasible that the cooling time and the flare time become equal for Doppler factors between 10 and 20. In this regime also, the pair opacity can be reasonably low. Thus it is possible that the IRB opacity estimated by Guy et al. (2000) may be too high. However, in view of the various approximations used, these conclusions can only be regarded as tentative. However, they do point the way to more detailed models in which the various effects can be modelled in more detail. Such models should include detailed calculations of inverse Compton emission in anisotropic radiation fields and the calculation of the post-shock flow, taking into account both particle acceleration and relativistic fluid dynamics.

\subsection{Relationship to Numerical Simulations of Relativistic Jets}

Our approach to modelling shocks in relativistic jets that we have described above is analytic and one can ask, is there any point in such a treatment given the impressive simulations of relativistic jets that have been carried in recent years? (For an introduction to the literature on this work one may consult the recent papers of Agudo et al. (2001), Rosen, Hughes, \& Duncan (1999), and Komissarov \& Falle (1997).) The answer is yes for several reasons:

1. Analytic/semi-analytic approximations of jet dynamics are useful when modelling blazar emission and for other analytic estimates of jet behaviour. Simple models (provided they capture the relevant physics) streamline the process of model fitting.

2. The numerical simulations are extremely useful for the purpose for which they were intended, e.g. in comparing relativistic and non-relativistic jet stability (Rosen et al. 1999) or in a qualitative assessment of the behaviour of superluminal components 
(Agudo et al. 2001). However, for computational reasons related to the Courant criterion, the jet densities of the simulations do not approach the low values that we expect of relativistic jets in AGN. To be specific, consider a jet in which the relativistic electron distribution has a lower cutoff at a Lorentz factor of $\gamma_{\min }$; let the pressures of the jet and interstellar medium (ISM) be $p_{\text {jet }}$ and $p_{\text {ISM }}$ respectively, and let $T_{\text {ISM }}=10^{7} T_{7}$ be the temperature of the ISM; let $a$ be the electron spectral index. Then the ratio of jet electron number density to ISM electron number density is given by

$$
\begin{aligned}
\frac{n_{\mathrm{e}, \mathrm{jet}}}{n_{\mathrm{e}, \mathrm{ISM}}} & \approx 6\left(\frac{a-2}{a-1}\right) \gamma_{\min }^{-1}\left(\frac{p_{\mathrm{jet}}}{p_{\mathrm{ISM}}}\right)\left(\frac{k T}{m_{e} c^{2}}\right) \\
& \approx 1.6 \times 10^{-3} \gamma_{\min }^{-1} T_{7}
\end{aligned}
$$

for a jet in pressure equilibrium with its surroundings and $a=2.2$. Therefore, for an electron-proton jet and an ISM temperature $\sim 10^{7} \mathrm{~K}$, the ratio of densities is of order $10^{-5}$ since $\gamma_{\min } \sim 100$ (Bicknell et al. 2001). For an electron-positron jet, $\gamma_{\min } \sim 10-100$ and the ratio of mass densities $\sim 10^{-8}$. The only simulations that begin to approach these remarkable densities are those of Agudo et al. (2001), in which the ratio of mass densities is $10^{-3}$. Of course one can learn a lot from simulations that depend mainly upon the ordering of densities rather than the absolute values. However, such simulations do not give a good idea of the interaction of the jet with the external medium since this strongly depends upon the jet density ratio.

3. It is feasible that one can utilise numerical simulations to understand internal jet dynamics in a jet in which the internal energy and the mass density are dominated by relativistic particles if the parameter

$$
\chi=\frac{\rho c^{2}}{4 p} \lesssim 1
$$

where $\rho$ is the rest mass density (Bicknell 1994, 1995). For the low density simulations of Agudo et al. (2001)

$$
\begin{aligned}
\chi & =\frac{\rho_{\text {jet }} c^{2}}{4 p_{\text {jet }}}=\frac{\rho_{\mathrm{ISM}} c^{2}}{4 p_{\mathrm{ISM}}}\left(\frac{\rho_{\mathrm{jet}}}{\rho_{\mathrm{ISM}}}\right)=\frac{\mu m_{p} c^{2}}{4 k T_{\mathrm{ISM}}}\left(\frac{\rho_{\mathrm{jet}}}{\rho_{\mathrm{ISM}}}\right) \\
& \approx 1.6 \times 10^{5} T_{7}^{-1}\left(\frac{\rho_{\mathrm{jet}}}{\rho_{\mathrm{ISM}}}\right) .
\end{aligned}
$$

For the parameters of the Agudo et al. (2001) simulation, $\chi \sim 100$. For one thing, this means that the velocities of internal shocks are not relevant to blazar emission regions in such a model.
One could put several counter-arguments to the above. For example, the interstellar medium temperature could be higher. But then one would have to reconcile this with the lack of observed high temperature bremsstrahlung from AGN. In summary, the current simulations are impressive and the various groups involved have boldly moved into a previously uncharted region of parameter space. However, the simulations do not currently address the physics of blazars. Clearly that is a challenge for the future.

\section{Acknowledgments}

We thank the organisers of this workshop for a stimulating meeting. SJW wishes to thank the organisers and the Heidelberg Sonderforschungsbereich for travel support. We acknowledge constructive criticism of the referee that contributed to an improved version of this paper.

\section{References}

Agudo, A., Gómez, J.-L., Martì, J.-M., Ibánez, J.-M., Marscher, A. P., Alberdi, A., Aloy, M.-A., \& Hardee, P. E. 2001, ApJ, 549, L183

Bicknell, G. V. 1994, ApJ, 422, 542

Bicknell, G. V. 1995, ApJS, 101, 29

Bicknell, G. V., Wagner, S. J., \& Groves, B. A. 2001, in The Oxford Radio Galaxy Workshop, ASP Conference Series 250, eds R. A. Laing, \& K. Blundell (San Francisco: ASP), 80

Blandford, R. D. 1994, in The First Stromlo Symposium: The Physics of Active Galaxies, ASP Conference Series 54, eds G. Bicknell, M. Dopita, \& P. Quinn (Provo, Utah: ASP), 23

Blandford, R. D., \& Levinson, A. 1995, ApJ, 441, 79

Ghisellini, G. 1997, in Relativistic Jets in AGNs, eds M. Ostrowski, M. Sikora, G. Madejski, \& M. C. Begelman (Krakow: Poligrafia Inspektoratu Towarzystwa Salezjanskiego), 262

Guy, J., Renault, C., Aharonian, F. A., Rivoal, M., \& Tavernet, J. 2000, A\&A, 359, 419

Komissarov, S. S., \& Falle, S. A. E. G. 1997, MNRAS, 288, 833

Lamer, G., \& Wagner, S. J. 1998, A\&A, 331, L13

Landau, L. D., \& Lifshitz, E. M. 1987, Fluid Mechanics, 2nd English Edition (Oxford: Pergamon)

Liang, E. P. T. 1977, ApJ, 211, 361

Mastichiadis, A., Georganopoulos, M., \& Kirk, J. G. 2001, in High Energy Gamma-Ray Astronomy, eds F. Aharonian, \& H. Voelk (New York: American Institute of Physics), 688

Rees, M. J. 1978, MNRAS, 184, 61P

Rosen, A., Hughes, P. A., \& Duncan, G. C. 1999, ApJ, 516, 729

Svensson, R. 1987, MNRAS, 227, 403 